\title{
libEnsemble: A Library to Coordinate the Concurrent Evaluation of Dynamic Ensembles of Calculations
}

\author{
Stephen Hudson, Jeffrey Larson, John-Luke Navarro, and Stefan M. Wild \\ Argonne National Laboratory \\ Mathematics and Computer Science Division \\ \{shudson, jmlarson, jnavarro, wild\}@anl.gov
}

\begin{abstract}
Almost all applications stop scaling at some point; those that don't are seldom performant when considering time to solution on anything but aspirational/unicorn resources. Recognizing these tradeoffs as well as greater user functionality in a near-term exascale computing era, we present libEnsemble, a library aimed at particular scalability- and capability-stretching uses. libEnsemble enables running concurrent instances of an application in dynamically allocated ensembles through an extensible Python library. We highlight the structure, execution, and capabilities of the library on leading pre-exascale environments as well as advanced capabilities for exascale environments and beyond.
\end{abstract}

\section{Introduction and libEnsemble Aims}

Scientific calculations are able to use increasing amounts of computational resources. Even so, the calculation's time to solution will eventually stop improving as additional resources are used. Many applications would still want to efficiently use resources such as emerging exascale computers; running concurrent instances of the calculation is a natural next step for goal-oriented application problems.

We refer to such instances of calculations for a shared goal as members of an ensemble. When considering computations from an ensemble-based view, many tradeoffs arise. For example, some algorithmic approaches may scale to far greater concurrency than others. A case of the former is brute-force computations (e.g., numerical integration by random sampling or by evaluating a deterministic low-discrepancy sequence): given an ensemble size corresponding to the concurrency level, the members of the ensemble can be formed a priori into a predetermined set and then be given out for evaluation. On the other hand, for many performance measures other than utilization or in less-than-ideal settings (e.g., when the calculations show nontrivial variability in their individual runtimes, when calculations may fail in a nonuniform way), such a brute-force ensemble approach is clearly unsatisfactory. That is, even if the resulting computation scales (near-)perfectly with ensemble size, the baseline (ensemble size of one) case is so poor as to render such naturally scalable approaches inferior for imagined concurrency levels. At the other extreme are entirely sequential approaches, which assume that a calculation is saturating available computational resources and that subsequent calculations may be optimally determined based on the result of all prior calculations.

In this paper we introduce libEnsemble [1], a Python library that seeks to empower users to address such tradeoffs. libEnsemble originated in the confluence of 
- designing outer-loop optimization, sensitivity analysis, and uncertainty quantification solvers around PETSc $[2,3]$ capabilities;

- exploring high-performance Python functionalities such as petsc4py ${ }^{1}$ and mpi4py [4]; and

- developing scalable implementations of APOSMM.

The latter represents an asynchronous multistart optimization method [5,6] that requests points either randomly generated or arising in various local optimization runs. In most use cases, these points parameterize computational simulations run on HPC systems at Argonne.

Additional design motivations included consideration of cases where single instruction, multiple data (SIMD) workflows may hamper meaningful progress toward a stated goal. As an example, deep/supervised machine-learning methods often presume that a fixed batch size of training data is used in every iteration. Such methods are rooted in resource-constrained settings where model evaluations naturally exploit SIMD benefits. Constraining oneself to such a workflow may result in more iterations, instructions, or data accesses than approaches where a batch size is determined adaptively $[7,8]$.

The design aims of libEnsemble include the following:

1. Tightly coordinated communication between a small number of highly configurable functions

2. Ability for resource-aware ensemble execution

3. Significant control over output and future ensemble members

4. Extreme portability, targeting automated scaling from laptops to exascale systems

Below we expand on these points and outline this paper.

In order to tightly coordinate parallel ensembles of calculations across a variety of scales and communication media, libEnsemble employs a basic manager-workers paradigm. As detailed in $\S 2.1$, workers call Python generator and simulator functions (or wrappers) to perform any type of computation and exchange data with the manager to determine and initiate future ensemble members. Such a paradigm enables the support of multiple communication protocols (§3.2). Generator, simulator, and allocation functions together are user functions (§2) and form the basis of all computations performed with libEnsemble.

To address the second aim, libEnsemble uses a manager to allocate work to the workers via a resource-aware allocation function $(\S 2.2)$ designed to enable ensemble evaluations that can adapt to available resources. The allocation of libEnsemble work to workers via an allocation function is facilitated by a history array (§3.3) of all pending and completed computations performed by workers via generator and simulator functions. This allocation can readily account for the availability of resources relative to history-based insights.

libEnsemble addresses scenarios where ensemble members need to be dynamically defined and controlled. Classical ensemble-based computations include linear system solvers involving multiple right-hand sides. When a typical direct method is used for such multiple right-hand-side problems, a single (e.g., factorization-based) expense is paid in order to benefit the concurrent solution for all possible right-hand sides. Such a direct solution method is a powerful technique that can be deployed for many complex problems and modern computational science problems (see, e.g., [9,10]). When the expense for effectively precomputing all possible ensemble solutions is implausible or

\footnotetext{
${ }^{1}$ See https://pypi.org/project/petsc4py/, which has subsequently been incorporated in PETSc via the --with-petsc4py option
} 
unnecessary, obvious alternatives include iterative solvers, such as those deployed in PETSc [2] and Trilinos [11]. libEnsemble is especially designed with consideration for settings where there may be benefits from ensemble members using shared resources and data structures (e.g., discretizations shared among most members) without an assumption that each ensemble's calculation will share a common control flow. By not restricting ensemble members to rely on precomputed work, libEnsemble seeks to enable use cases where it is often better to focus on calculations in dynamically evolving configurations. In $\S 4$ we overview several such use cases, ranging from parameter space exploration with simulation failure indicators to design optimization to statistical calibration. In each case, we assume that, by itself, an ensemble member may not be able to fully benefit from its available resources and hence it is important to concurrently execute ensemble members.

One obstacle to employing libraries addressing extreme-scale concurrency afforded by supercomputers is the inability to develop and deploy such capabilities at intermediate levels, including a user's laptop. libEnsemble was developed as part of the software ecosystem [12] for the U.S. Department of Energy exascale computing project (ECP) and with a Python-based front end for use on many systems. libEnsemble readily allows user functions to launch and interface with applications on high-performance hardware ( 55 ). libEnsemble's executor can dynamically split allocated resources (including GPUs) among workers to the user's specifications and interface with emerging workflow schedulers such as Balsam [13]. On the other hand, libEnsemble is available as a PyPI (pip), Conda, and Spack package. In all such forms, libEnsemble is actively developed to be interoperable with other exascale-focused software technologies and is the first Python package incorporated in xSDK, the Extreme-scale Scientific Software Development Kit [14].

libEnsemble is one of a number of extreme-scale workflow software packages (e.g., RADICALEnsemble Toolkit [15] and other packages in the ExaWorks project [16]). libEnsemble primarily distinguishes itself via its generator-simulator paradigm that sidesteps requiring users to define task dependencies in favor of data dependencies between configurable Python user functions; see $\S 2$. This allows the user to focus their attention on function logic. This composable design also lends itself to exploiting the large library of example user functions that are provided with libEnsemble, maximizing code re-use. For example, users can easily select an existing generator function while modifying a simulator function for their specific use case. While libEnsemble provides a complete ensemble toolkit including a task executor interface, its modular design also allows users to plug in components from other workflow packages such as Parsl [17], resource management libraries like Flux [18], or various pilot systems.

libEnsemble is a growing software package; we highlight current and future development ideas in $\S 6$.

\section{2 libEnsemble User Function Overview}

An early part of libEnsemble's design was the decision to divide ensemble steps into generator and simulator routines as an intuitive way to express problems and their inherent dependencies.

libEnsemble was consequently developed to coordinate ensemble computations defined by

- a generator function that produces simulation inputs,

- a simulator function that performs and monitors simulations, and

- an allocation function that determines when (and with what resources) the other two functions should be invoked. 


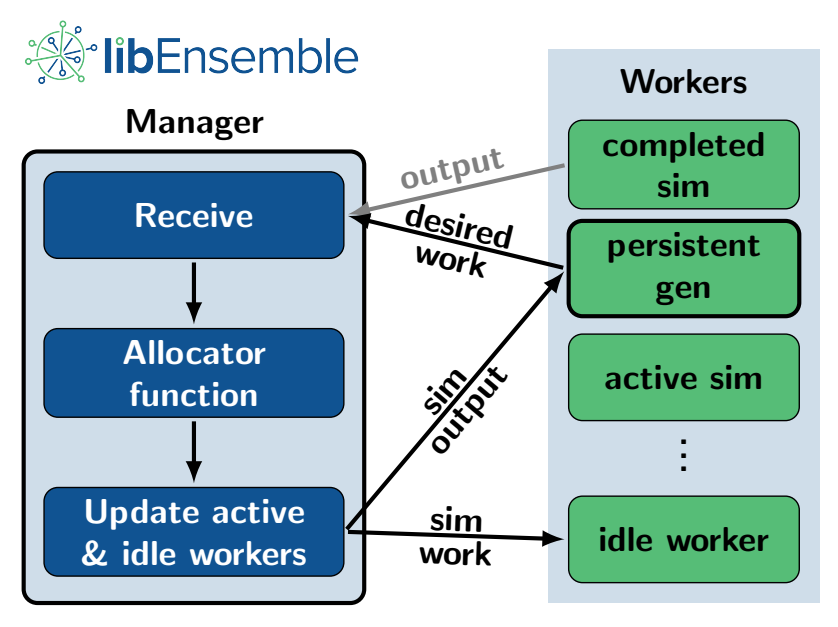

Figure 1: Example of data movement between libEnsemble's manager and workers. Here, the manager receives output from a completed simulation and also some other work requested by a persistent generator. This is given to the allocation function, along with information about what workers are active or idle. The allocation function determines what work should be done and with what resources.

Since each of these functions is supplied or selected by libEnsemble's users, they are typically referred to as user functions. User functions need not be written only in Python: they can (and often do) depend on routines from other languages. The only restriction for user functions is that their inputs and outputs conform to the user function API. Therefore, the level of computation and complexity of any user function can vary dramatically based on the user's needs.

An example of the interplay between these user functions is given in Fig. 1. In most libEnsemble workflows, users develop and combine a set of their own user functions or develop a single user function to fulfill a particular algorithmic purpose or support a specific application. In all cases, users are invited to browse examples distributed with libEnsemble and modify them for their own needs.

\subsection{Simulator and Generator Function Characteristics}

On being sent a unit of work by the manager, a worker calls the requested simulator or generator function with the following parameters:

- H: an assigned selection of the history array (see $\S 3.3$ )

- persis_info: a dictionary of per-worker persistent information

- sim_specs or gen_specs: a dictionary of configuration fields and data types

- libE_info: a dictionary of libEnsemble-specific information, typically used in persistent functions (see $§ 2.1 .1)$

Inside each function, input data is extracted from the history array selection, and an output array is defined based on the user-defined output data types for the function found in sim_specs or gen_specs. This array is populated by the simulator or generator routines. In the simple simulation function in Fig. 2, the output array is populated by matrix norms of input values. 


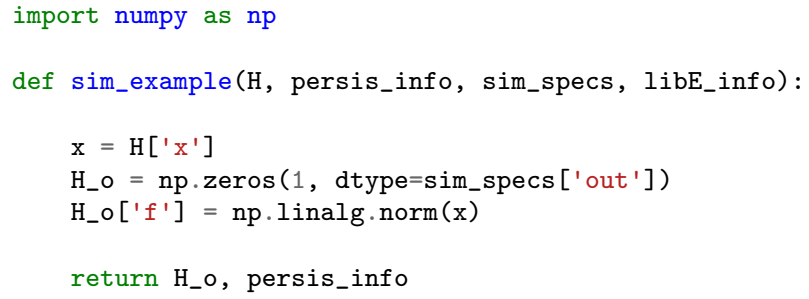

Figure 2: Simple simulator function. The output array is defined to match the expected 'out' specifications and then populated by calculations. This array is returned to the manager.

Any level and complexity of computation can be performed. Many functions use libEnsemble's executor (§3.6) to launch applications and monitor results. Other functions can be written to initiate parallel routines via Python's multiprocessing module or even detect whether alreadyscheduled simulations become unnecessary and request their cancellation.

Persistent information or structures for use across user function calls can be stored in persis_info. For example, Fig. 3 depicts a simple generator function that uses a NumPy random number stream that is stored in persis_info in order to generate random points. This stream can be used in future calls to the generator function if more controlled random number generation is desired.

Following computations, each simulator or generator function returns three items:

- The output NumPy array, populated with evaluations

- persis_info, to be passed back on the next function call

- An optional calc_status integer, typically for logging the status of application runs

\subsubsection{Persistent Functions}

For many more sophisticated generator or simulator functions, the paradigm of initializing, computing, and returning potentially hundreds of times over the course of a libEnsemble workflow can

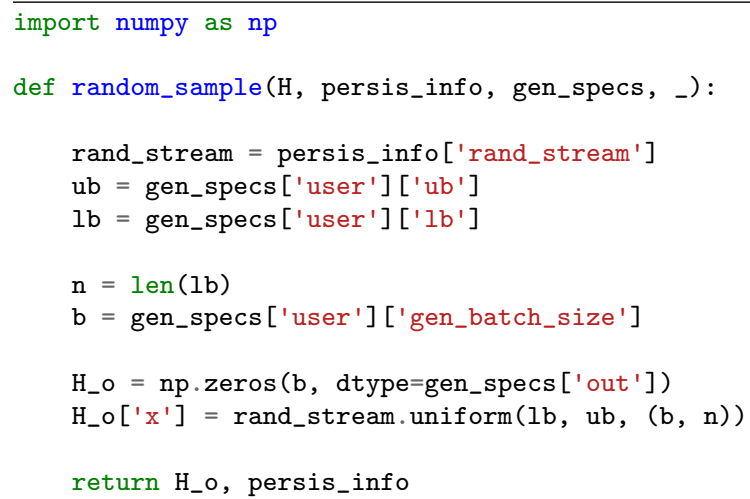

Figure 3: Example generator function. The random stream in persis_info is used to generate values and is returned to the manager for use in future calls. 


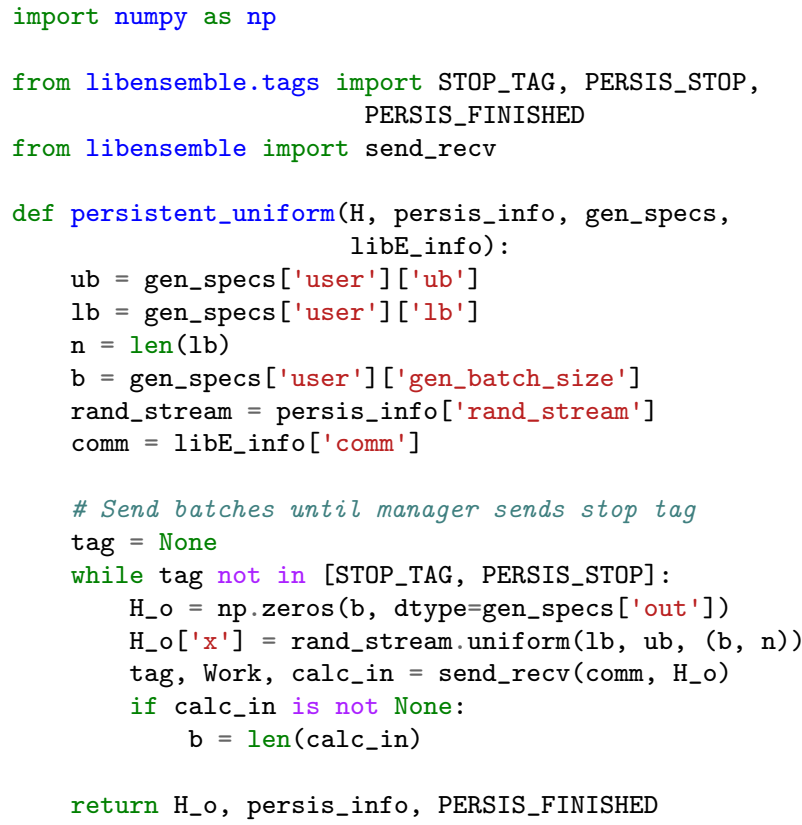

Figure 4: Example persistent generator function. The function generates values while communicating directly with the manager every loop.

be inefficient. An alternative approach involves structuring the function to receive work, perform computations, and return results in a loop, communicating directly with the manager instead of returning results to the calling worker. A simulator or generator function structured in this way is referred to as persistent, with the calling worker considered a persistent worker. Persistent functions take advantage of the communicator object included in libE_info and several helper functions to send and receive data from the manager.

An example persistent user function depicting the process of looping and directly communicating with the manager is shown in Fig. 4. Note that the same random uniform sampling is performed here as in Fig. 3, but the results are immediately sent to the manager via the send_recv() helper function. The manager communicates back a tag and additional work. Persistent functions compare tags from the manager to know when to exit.

The APOSMM parallel optimization generator function (discussed further in §4.1) included with libEnsemble is persistent so it can maintain and advance local optimization subprocesses based on the results of complete simulations. Previous nonpersistent versions of APOSMM had to reconstruct the sequence of local optimization runs on every call to the generator.

\section{$2.2 \quad$ Allocation Function Characteristics}

As opposed to the simulator and generator functions that are called by the workers, each allocation function is called by the manager as part of the main loop. The call includes a list of workers and their statuses, the current state of the history array, and the specifications available to both generator and simulator functions.

Allocation functions query the status of entries from the history array and map these to available workers, using a handful of helper functions to select and define units of work for chosen workers. This allocation is captured in a Work dictionary. The allocation function returns the resulting Work 
dictionary and persis_info to the manager. The manager will iterate over the Work dictionary and send entries to assigned workers.

For allocation functions, as with all user functions, the level of complexity can vary widely. Various scheduling and work distribution features are available in the existing allocation functions, including prioritization of simulations, returning evaluation outputs to the generator immediately or in batch, assigning varying resources sets to evaluations, and other methods of fine-tuned control over the data available to other user functions. libEnsemble also includes an allocation function that allows users to simply distribute pre-existing simulator work without needing a generator.

For persistent generator or simulator functions, the allocation function is responsible for initiating a given function as persistent and flagging that the associated worker is in "persistent mode."

\section{Configuring and Running libEnsemble}

Users have a large degree of control over the libEnsemble run and its components via a Python calling script, where libEnsemble's parallelism method, the history array, user-application registration, and other characteristics are defined.

\subsection{Calling Script}

Any given libEnsemble instance is configured and run from a Python script referred to as the calling script. The calling script defines parameters for the ensemble and specifies the user functions, followed by a call to the primary $\operatorname{libE()}$ initialization function, with each configuration dictionary passed in as parameters, as in Fig. 5. A libEnsemble calling script can be run by using an MPI runner (which requires mpi4py communications, see §3.2.1) or serially with either of the other two communication schemes described in $§ 3.2$.

libEnsemble does not require a specialized runtime environment or pilot program, so long as workers are able to issue applications directly (e.g., via an MPI runner). This is the case on standalone systems and many clusters or when libEnsemble is run on the dedicated launch nodes of many supercomputers. This keeps the user's installation and run requirements as simple as possible. In such circumstances, libEnsemble is initiated simply by running the calling script with Python:

\$ python libE_routine.py.

Because libEnsemble uses MPI by default, this statement produces only a single MPI process, leaving none for workers. Specifying enough processes is covered in the next section.

In scenarios where the direct launching of applications from workers is not possible or where more advanced scheduling schemes are desired, such as using multiple resource pools (on the same or different systems), libEnsemble can employ the Balsam (see §5.3) executor.

\subsection{Running libEnsemble}

libEnsemble supports three main methods for manager-worker communications upon initiation:

§3.2.1 A message passing interface (MPI) via mpi4py [4] with an MPI implementation such as MPICH2 [19]

§3.2.2 Python's multiprocessing module for local communications

§3.2.3 Distributed/cloud-based environments via the transmission control protocol (TCP) 


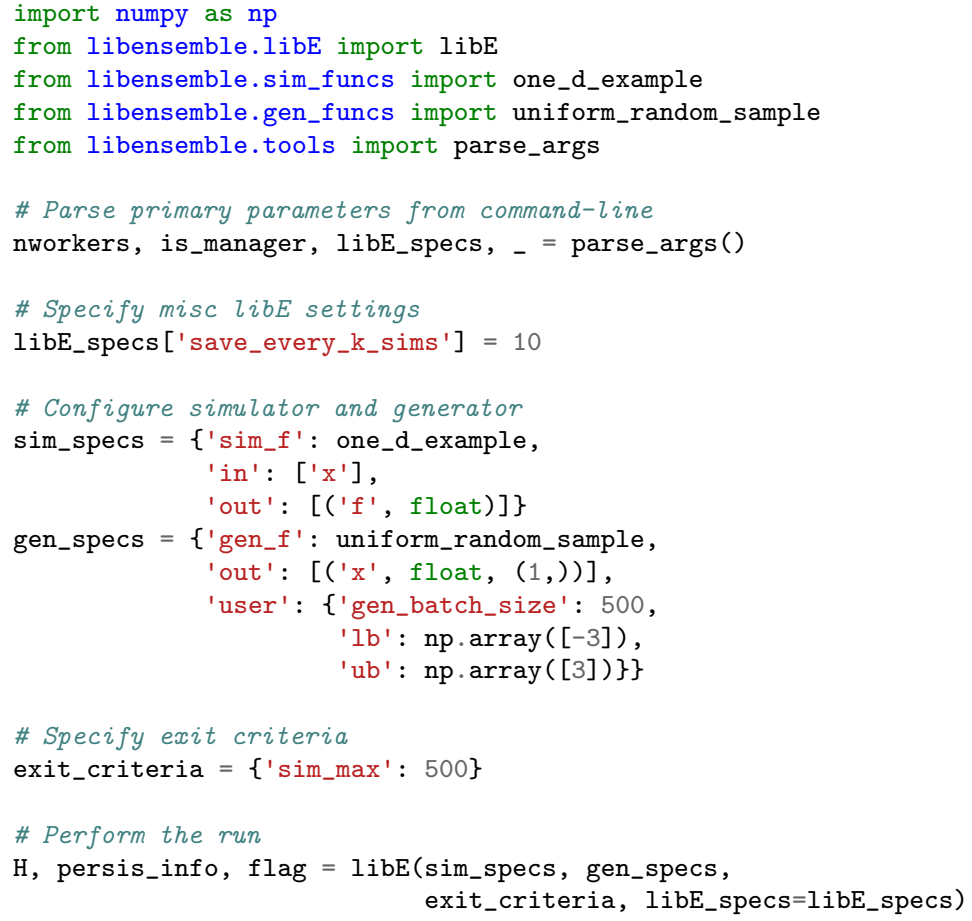

Figure 5: Example calling script from libEnsemble's regression tests. libEnsemble and the user functions are configured via dictionaries, each of which is passed to $\operatorname{libE}()$ to initialize libEnsemble. A complete list of options for these dictionaries is available on libEnsemble's online documentation [1].

Switching between these methods does not require workflow code changes, although choosing to use either MPI or local communications typically implies a preference for what nodes libEnsemble launches on and how processes are distributed. By default, libEnsemble launches with MPI.

\subsubsection{MPI}

Running libEnsemble with MPI-based communication potentially scales best when running with many workers on multinode systems, and it allows users to be flexible with how processes are distributed across nodes (see §5). libEnsemble with MPI is typically initiated via a single mpirun or equivalent statement with python, providing the number of workers to mpirun and the libEnsemble calling script as the primary argument to python. MPI rank 0 becomes the manager process, and the remaining become worker processes, for example:

\$ mpirun -n 64 python libE_calling_script.py.

Using the MPI executor module to invoke an MPI-based simulations (see §3.6) while running libEnsemble with MPI produces nested MPI processes. Nesting MPI is not supported by Open MPI but is supported by MPICH and its derivatives. Users who prefer Open MPI can overcome this circumstance by using a proxy application launcher such as Balsam. libEnsemble with MPI requires the installation of mpi4py [4]. 


\subsubsection{Multiprocessing}

The multiprocessing option uses Python's built-in multiprocessing module for manager/worker communications and is referred to as local mode. All of libEnsemble's manager and worker processes are initiated on a single node (libEnsemble must be launched in central mode); libEnsemble's executor is needed to use the remaining nodes in an allocation. On three-tier systems such as ALCF's Theta and OLCF's Summit, libEnsemble should be run on the launch nodes in local mode, allowing the entire compute node allocation to be available for application submissions via the executor.

An optional helper function for calling scripts allows users to run libEnsemble with multiprocessing from the command line:

\$ python libE_routine.py --comms local --nworkers 64.

\subsubsection{TCP}

Running libEnsemble with TCP communication allows the manager process to run on one system and workers to remote systems or nodes. The architecture for this approach is based on the Plumbing for Optimization with Asynchronous Parallelism (POAP) event-driven framework [20].

\subsection{History Array}

libEnsemble's manager maintains a NumPy structured array of all input and output values from each generator and simulator function call during a run. User functions must return their computations in NumPy arrays so that they can be slotted into this history array. The history array maintains sim_ids for each unit of work produced by a generator and worker IDs that record which worker was assigned to call an associated user function. The Boolean fields given and returned record whether a row in the history has been distributed for simulation and whether results have returned from a simulation, respectively.

The current state of the history array is returned to the user on concluding a successful libEnsemble run, or it is saved to file in the event of a fatal error or timeout. The maintenance of these fields and all computations allows users to restart libEnsemble routines in the event of a failure by providing a history array.

\subsection{Logging, Shutdown, and Error Handling}

libEnsemble logs ensemble routine information to two files by default. The ensemble.log file contains overarching run information such as available nodes, where managers and workers are initiated, and the status of applications launched via the executor. libEnsemble's logger uses the standard Python logging levels of DEBUG, INFO, WARNING, and ERROR, with INFO being the default level. libEnsemble also uses an additional custom MANAGER_WARNING level between WARNING and ERROR. The logging level can be adjusted in the calling script.

In libE_stats.txt, a line is appended on the conclusion of each user function call, containing information on the calling worker, what type of function was called, duration, and the status of any launched applications.

The libEnsemble shutdown process, followed in the case of a standard exit or an exception, aims to extract and save outstanding data where possible, to exit cleanly in different scenarios, prevent hangs, and to maintain the manager's role in coordination, decision making, and logging. The manager captures and handles exceptions that occur both in the manager code itself and 
exceptions that originate on workers. Worker exceptions are caught and communicated to the manager, which can then report the exception and determine how to handle the error. The default handling is to issue stop signals to all workers, dump the history and state information to file, and close down the ensemble.

\subsection{Output and Working Directories}

libEnsemble's output and working directories are highly configurable via options specified in the calling script. Workers can perform each of their computations in a single ensemble directory or switch into unique sub-directories. Each output directory can be set to contain copies or symbolic links of other files or directories; this is helpful for users with applications that may modify input files or cases where large initialization files need not be copied for each simulation evaluation. Such configurability allows users to take advantage of high-speed disks, configure the location of checkpoint data, and prevent conflicting I/O in their ensemble calculations.

On the conclusion of a libEnsemble routine, workers can copy back their results to the location that libEnsemble was originally launched from (usually a user's project directory).

\subsection{Support for User Applications}

libEnsemble aims to offer a portable framework for users to provide Python-based functions describing their desired workflow. This includes the capacity of each worker to launch and manage user applications in a way that is agnostic to schedulers, MPI runners, launch constraints, and other system variants.

The libEnsemble executor provides the user a portable interface to express an application submission. The executor subpackage is provided with a base executor class. If the base class is used directly, it will simply subprocess serial applications locally. The MPIExecutor subclass is most commonly used, which has an extended interface. Most of the following details are based around this subclass; alternative executor subclasses may easily be added.

The executor can utilize libEnsemble's detection of resources and features launch, polling, and termination mechanisms. Launch retries are automatically used in the case of failures. This interface forms one part of the separation of concerns between the user's ensemble specification and the mechanism of launching and managing applications. For example, a completely different launch system can be swapped out by registering a different executor in the calling script. One of these, included in the libEnsemble package, is the Balsam executor BalsamMPIExecutor (described in $\S 5.3)$, which can be used in scenarios where the direct launching of MPI applications from the workers is infeasible.

While libEnsemble employs autodetection for schedulers, node lists, MPI runners, and system factors, there is also a means for the user to provide this information, overriding detection.

The provision of multiple manager/worker communication substrates, along with alternative executors, means that most run scenarios can be handled with minimal changes to the user scripts.

\section{Use Cases and Examples}

Development work for libEnsemble can take multiple forms. Some researchers are looking to develop efficient generators based on state-of-the-art numerical algorithms with no specific simulator in mind. Others have a simulator (and sometimes a scientific or engineering aim) in mind and are looking to test or interface with generators. 


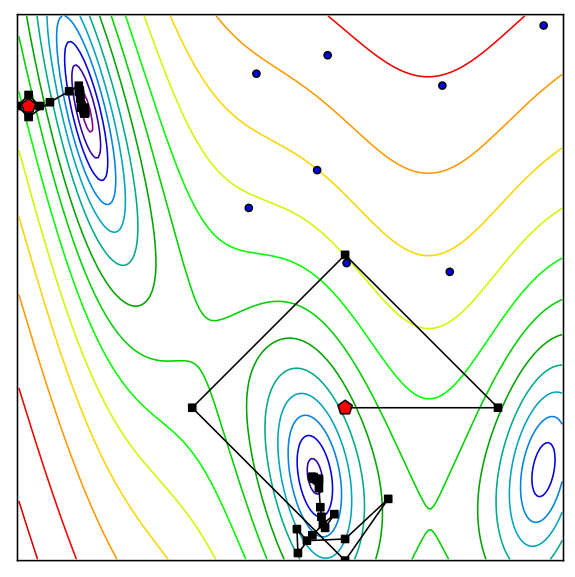

Figure 6: Example of the APOSMM generator. Random samples with better points in their neighborhood are marked as blue circles; red pentagons show random points that start runs; and black squares are points arising from local optimization runs.

Here we review four early uses of libEnsemble, each with a particular perspective/objective: implementing a generator based on a parallel multistart optimization algorithm ( $\$ 4.1)$, killing a simulation based on its intermediate output ( $\$ 4.2$ ), running multi-GPU simulations to achieve a goal within a limited time budget $(\S 4.3)$, and efficiently calibrating a computer model by removing queued calculations before they are executed (\$4.4).

\subsection{Parallel Multistart Simulation-Based Optimization}

Many computationally expensive simulations of scientific phenomena have nonlinear, nonconvex behavior with respect to their inputs. In such cases, performing numerical optimization can be difficult since the performance of local optimization methods can depend heavily on starting values and global optimization methods can require the evaluation of undesirably many simulations.

APOSMM [5] is an optimization solver for finding multiple minima using asynchronously parallel objective evaluations. APOSMM accepts or performs an initial sample of the parameter space and starts local optimization runs from points that do not have better points in their respective neighborhood. This neighborhood is adjusted dynamically as outputs from more sets of parameters are observed. Fig. 6 shows the contours of a test objective function and the points that APOSMM has requested at one snapshot in time.

In libEnsemble, APOSMM requires the user to declare a local optimization method, with example interfaces provided for SciPy, NLopt [21], and PETSc/TAO methods, as well as any external routine. That is, the local optimization routine need not be in Python, and the libEnsemble regression tests provide examples of such functionality.

As a generator function, APOSMM allows users to control many aspects of optimization including tolerances and settings for their local optimization method and how many concurrent local optimization runs are progressing at a given time. The asynchronous nature of APOSMM prevents blocking on a simulation output from potentially unrelated runs: as soon as a given run's simulation output is returned from the manager, the next point in that run can be given. This allows APOSMM to efficiently utilize computational resources. 


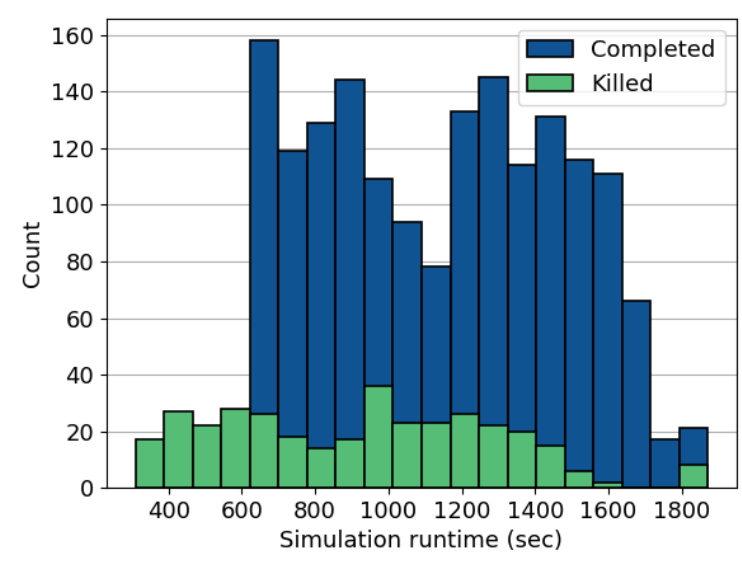

Figure 7: Runtime results for OPAL simulations of the LCLS-II beamline photoinjector in an initial sampling of the 2,046 parameter values. Although this routine used 1,023 workers, killing runs saved considerable computational resources. The average time for the 1,696 completed simulations was 1,150 seconds, whereas the average time for the 350 killed simulations (the completed output of which are not useful) was 941 seconds.

\subsection{Preemption of Photoinjector Sample Configurations}

Researchers at the SLAC National Accelerator Laboratory use OPAL [22] to simulate the photoinjector for the LCLS-II free-electron laser beam. They are looking for configurations of laser, magnet, and RF settings that produce a beam with desired properties [23].

A typical first step involves sampling over a large domain of allowable configurations before refining the domain to target specific regions of parameter space. The OPAL simulator reports when particles are lost from the beamline (an untenable situation in this use case) but does not support termination as soon as particles are lost. In the libEnsemble simulation function implementation, a feature was added to allow the libEnsemble worker to kill its calculation based on the contents of the OPAL output file. Figure 7 shows the benefit of being able to kill certain simulations. (The killing of simulations is managed by the libEnsemble executor; see §3.6.)

In this example, the ability to kill certain simulations based on the output in log files decreased the computational cost required for running this large collection of simulations. Figure 7 shows that, on average, simulations where particles were lost were killed 3.5 minutes earlier than the average run times for simulations for which particles were not lost.

\subsection{Designing Plasma-Based Accelerator Stages}

Plasma-based technology is a promising technology to accelerate and focus electron beams with a smaller device footprint [24]. Designing efficient plasma-based accelerators includes the tuning of focusing lenses after each plasma stage. Beamline scientists must determine lens properties, such as longitudinal position and strength correction factors, in order to optimize beam-quality metrics such as emittance.

High-fidelity simulations of such accelerator designs are critical to determining realizable designs, but each simulation requires the numerical solution of both electromagnetic fields and particle movement. WarpX [25] is a particle-in-cell $\mathrm{C}++$ code being developed under the DOE's ECP to address these challenges on emerging exascale platforms. 


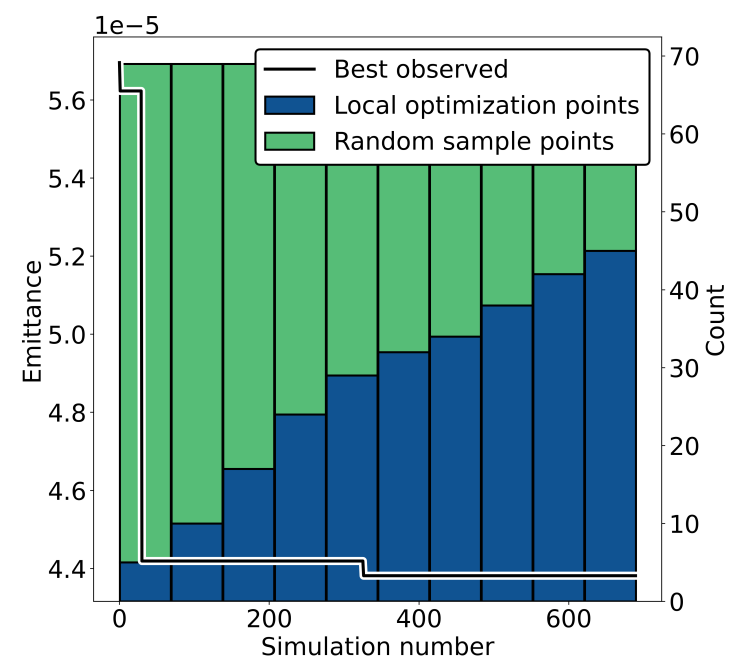

Figure 8: Progress of an APOSMM generator in minimizing the emittance from a beamline. Each simulation is a WarpX run on 12 Volta GPUs. The right vertical axis represents a visualization (with simulations aggregated into 10 bins) of the evolving proportion of simulations run for samplingbased exploration versus those run for local optimization refinement.

Here we illustrate the application of libEnsemble to facilitate multi-GPU WarpX-based simulations. As a case study we consider the tuning of two lenses separating three consecutive plasma stages. A GPU-optimized version of WarpX [26] was used to simulate each design in a $192 \times 192 \times 7680$ grid with 50,000 particles. This resulted in a typical simulation runtime of 25 minutes on 2 nodes of the OLCF Summit system (each node contains 6 NVIDIA Voltas).

Figure 8 shows the evolution of an emittance-based objective function value using 472 -node (12 Voltas) workers. We use an APOSMM-based generator that consists of both random sampling (for exploration of the design space) and local optimization (for refinement within particular regions of the design space) with the BOBYQA algorithm [27]. We see that as the optimization progresses, a larger number of the WarpX evaluations arise from concurrent local optimization runs and hence refining candidate solutions. By changing generator and allocation functions, in addition to resources such as the number of workers, libEnsemble can enable efficient use of connected resources such as architectures where performance is due primarily to GPU-based computation.

\subsection{Statistical Calibration of Simulation Models}

A common motif for capability-enhancing extreme-scale computing is the calibration of a numerical simulation model to data. Such problems arise throughout science and engineering and take forms ranging from inverse problems to supervised machine learning. Statistical calibration seeks model parameters in cases where the data or model are uncertain, and it tends to require many evaluations of the model.

One way to solve such problems is to use a Gaussian process (GP) [28] emulator to approximate the model output. A GP emulator can be constructed by evaluating the model at a well-designed, predetermined set of parameter values. This is a standard way to construct an emulator, in part because the process of evaluating a predetermined set of parameter values scales naturally up to the size of the set. In cases where such evaluations cannot be performed entirely in parallel, 


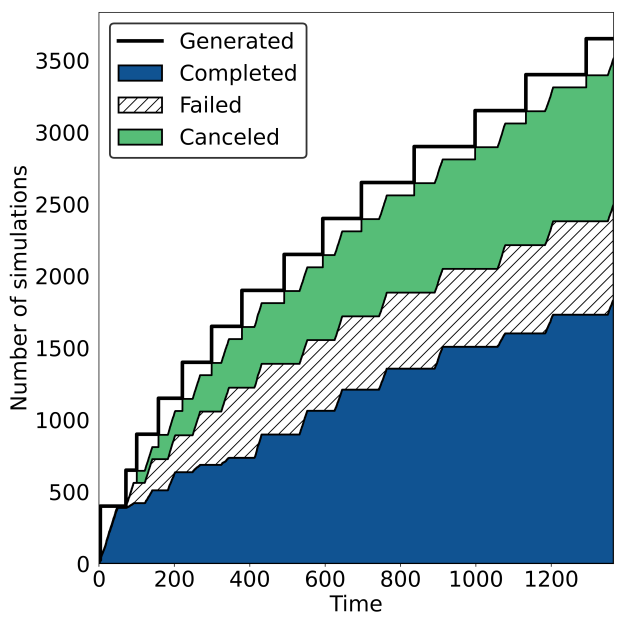

Figure 9: Number of borehole simulations that have been generated, completed, canceled, or marked as failed over the first 14 batches when solving a statistical calibration problem. Note that this generator function does not mark points as canceled until right before it produces its next set of generated points.

however, determining parameters to evaluate dynamically can lead to better emulator properties. Focusing evaluations on portions of a parameter space based on information obtained from previous evaluations can significantly improve emulator quality in parameter regions of particular interest for model calibration [29-31].

An active area of research in this area concerns dynamically determining batches of evaluations to aid the goal of calibration. Unsurprisingly, the larger the batch size (and thus potentially more parallelizable the evaluations), the more likely such evaluations are not best suited for the overall calibration goal. Determining a batch of evaluations becomes both more complex and more promising, in terms of overall time to solution, when considering a capability to preempt/cancel queued evaluations. One libEnsemble use case involves irregular communication patterns between generator and manager to preempt specific simulation evaluations. This includes the emulation capabilities in surmise [32,33] predicated on generator-directed (e.g., surmise-directed) cancellation of simulations.

As an illustration of such a use case, we consider a popular statistical test function involving the calibration of a water flow model based on observed data in a set of boreholes [34]. This test function requires little time to evaluate, and hence the simulation time can be easily artificially changed to benchmark a variety of difference computational paradigms.

Figure 9 shows the result of the statistical calibration generator run in persistent mode and creating batches of input values for evaluation. After an initial batch has been processed, the generator loops, receiving simulation results and, based on particular criteria, decides when to update the model, create new inputs for evaluation, or request cancellation of previously issued evaluations that are determined to no longer be useful. When evaluations are marked for cancellation, they may be in several states. If the evaluation has not yet been issued to a worker, it will not be sent. If it is already being processed by a worker, a kill signal will be sent by the manager. The worker can receive this signal and kill a running application (via the executor). The generator can also handle evaluations that were returned prior to registering for cancellation. 


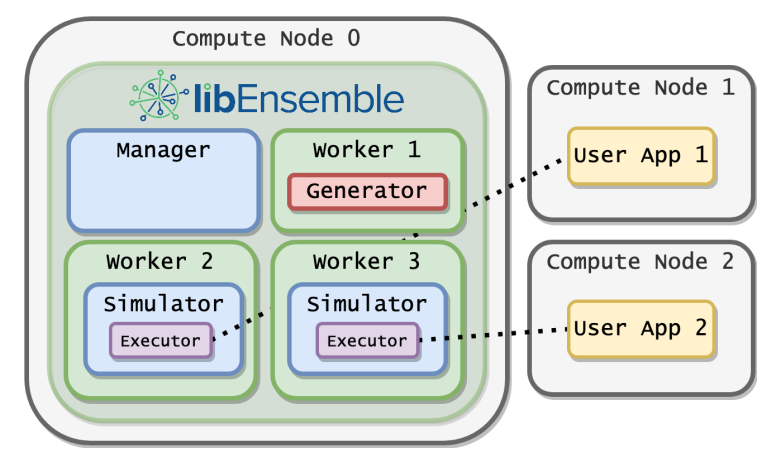

Figure 10: Process distribution when launched in central mode.

\section{Running on HPC Systems}

libEnsemble is developed, supported, and tested on systems of highly varying scales, from laptops to thousands of compute nodes. The aim is to allow the user, through a minimal set of configuration options, to easily run an ensemble in the optimal way for the target platform and available resources.

For example, libEnsemble may be run on launch nodes or compute nodes of high-performance computing (HPC) systems or remotely via TCP; it may use direct or proxy launch of user applications; and it may schedule resources to workers directly or allow the system to handle submitted tasks. libEnsemble also simplifies the process by automatically detecting and interfacing with schedulers (including Slurm, LSF and Cobalt), MPI runners (including mpirun, aprun, srun, jsrun) and node resources (via environment variables or transparently running a probe task).

\subsection{Configuring the Run}

On systems with a job scheduler, libEnsemble is typically run within a single job submission. All user functions will run on the nodes within that allocation. On multinode systems, there are two basic modes of configuring libEnsemble to run and launch tasks on the available nodes.

The first mode we refer to as the central mode, where libEnsemble's manager and worker processes are grouped on one or more dedicated nodes. Workers launch tasks onto the remaining allocated nodes, as in Fig. 10. Alternatively, in distributed mode, the libEnsemble (manager/worker) processes will share nodes with submitted tasks. This enables libEnsemble, using the mpi4py communicator, to be run with the workers spread across nodes so as to be co-located with their tasks, as in Fig. 11.

Configurations with multiple nodes per worker or multiple workers per node are both common use cases. The distributed approach, for example, allows the libEnsemble worker to read files produced by the application on local node storage. HPC systems that allow only one application

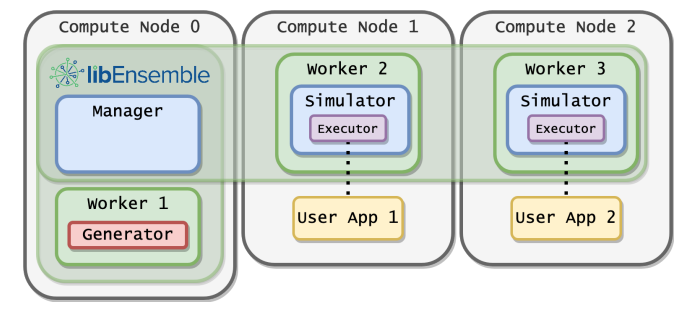

Figure 11: Process distribution when launched in distributed mode. 
to be launched to a node at any one time prevent distributed configuration.

libEnsemble has built-in resource management that will automatically detect the nodes available on most systems. Alternatively, the user can provide a node_list file in the run directory. While the resource manager will divide the nodes evenly to each worker by default, more dynamic options are available ( 55.5$)$. If the central mode option is set, then any node that is running a libEnsemble manager or worker will be removed from the node-list available for worker-launched tasks, ensuring that libEnsemble has dedicated nodes for user calculations.

libEnsemble is packaged with example submission scripts, configured to run either centrally or distributed, for a number of different systems and schedulers.

\subsection{Systems with Dedicated Launch Nodes}

Some large systems such as Summit, Sierra, and Theta have a 3-tier node setup; they have a separate set of launch nodes (e.g., MOM nodes on Cray Systems), which host user batch jobs or interactive sessions. Such systems provide a specialized MPI runner (e.g., aprun, jsrun) that has some application-level scheduling capability. MPI applications can be submitted only from these nodes.

There are two ways of running libEnsemble on these kinds of systems. The first, and simplest, is to run libEnsemble on the launch nodes. This is often sufficient if the workers' simulator and generator functions are not doing too much work (other than launching applications). This approach is inherently centralized, with the entire node allocation available for the worker-launched tasks.

To run libEnsemble on the compute nodes of these systems requires an alternative mechanism (e.g., Balsam, §5.3) for workers to launch tasks. Running libEnsemble on the compute nodes is potentially more scalable and will better manage simulator and generator functions that contain considerable computational work or I/O.

\subsection{Integration with Balsam}

Balsam is an HPC workflow management system that comprises a task database (e.g., using PostgreSQL), a pilot job launcher that pulls and executes new tasks in real time, and an edge service that can submit new batch sessions and transfer data between systems.

In the typical workflow, Balsam is initiated in the batch submission, where it is linked to an activated database and adds libEnsemble as the first task. Once running, libEnsemble can import the Balsam executor (in place of the regular executor), and all worker-submitted tasks will be routed to the Balsam database. Balsam will take on responsibility for task queuing and scheduling of resources to each task.

Balsam allows the libEnsemble package to be run on compute nodes of systems such as Theta, thus providing dedicated resources for running any expensive operations or $\mathrm{I} / \mathrm{O}$ and ensuring a

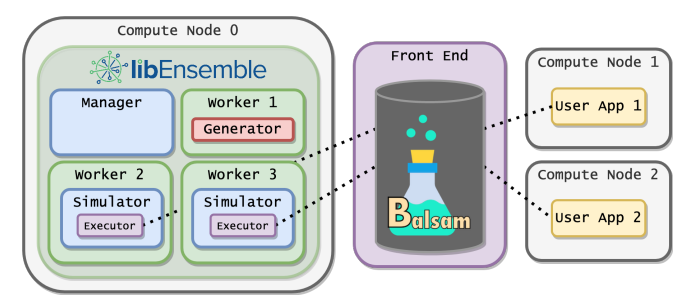

Figure 12: libEnsemble can submit tasks to a Balsam instance running on an intermediate launch node. Balsam dynamically schedules and launches these tasks to the remaining compute nodes. 
consistent architecture for any compiled applications run directly in the user functions. If using the MPI communicator, libEnsemble's workers can be spread over multiple nodes. Another advantage is that MPI tasks are decoupled from the parent communicator, so nested MPI support is not required.

The Balsam edge service component is not necessary for this scenario, but it provides a capability to automate the orchestration of data transfers between systems and the allocation of resources/job submission on those systems. This could be employed to run user tasks in different allocations and across different systems.

\subsection{Resource Management}

libEnsemble has built-in resource management. This entails the detection of system resources and the allocation of resources to workers.

The necessity for this sort of resource management is system dependent. Some systems, especially leadership-class supercomputers, support application-level scheduling by default. For example, the aprun command on Theta will find available nodes within the user's job allocation [35].

Some application schedulers, such as the jsrun system on Summit, support queuing of applications until a slot becomes available [36]. In this case, the user can easily oversubscribe application runs to the provided resource allocation.

Furthermore, libEnsemble has the option of submitting via Balsam, which can also queue and schedule the submitted tasks.

Many systems, however, will simply schedule applications to the same node(s) unless node information is specified for each application run. Furthermore, if working at a subnode level (multiple workers submitting tasks to the same nodes), node partition information may be required by the worker.

For this reason, and to enable a general, portable approach to resource management, libEnsemble detects available nodes and cores. Each worker can then be assigned a node or nodes and, if necessary, a partition within a node. Automatic resource detection runs by default but can be disabled or overridden by user-supplied information.

\subsection{Variable-Resource Workers}

The resource manager defaults to an even division of resources amongst workers. However, more flexible and dynamic allocations are possible.

One common case is a generator that runs in place and does not require additional nodes/cores. For this scenario, especially if the generator runs in persistent mode, a worker may be specified as a zero-resource worker. The user can then add an additional worker to make use of those resources. In Figs. 10 and 11, the generator is a zero-resource worker.

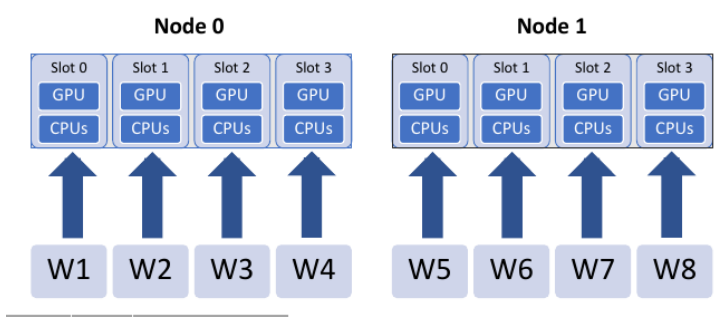

Figure 13: Initial fixed mapping of resources to workers. 

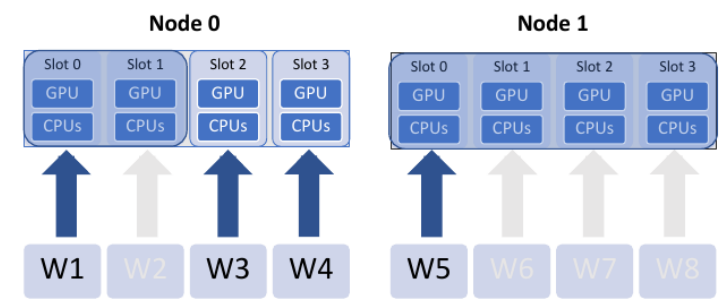

Figure 14: After the initial mapping, resources can be dynamically reassigned to workers.

Ongoing work seeks to update libEnsemble's resource management and allocation functions to provide better support for the dynamic scheduling of resources to workers. The current approach takes a fixed initial mapping of workers to resources, shown in Fig. 13, where the resources allocated to one worker are defined as a resource set. Both manager and workers are aware of this mapping.

Resources can then be dynamically reassigned to workers, at the granularity of resource sets as shown in Fig. 14. This feature supports subnode-level workers, in which case the allocation function's default scheduling policy will find the smallest partition on a single node. The local partition information is available to each worker, where it can be accessed directly in user functions or implicitly via the executor. An example of a simulation function accessing resources information to set the CUDA_VISIBLE_DEVICES environment variable is shown in Fig. 15. The number of resource sets required for each simulation can be determined in the generator user function.

This approach minimizes communication between the manager and workers. An alternative approach is for workers to remain unaware of the global mapping. In such a model, the manager can explicitly assign resources to workers, thus allowing for greater flexibility: partitions can be arbitrary, and it may be easier to manage multiple pools of resources, without synchronization.

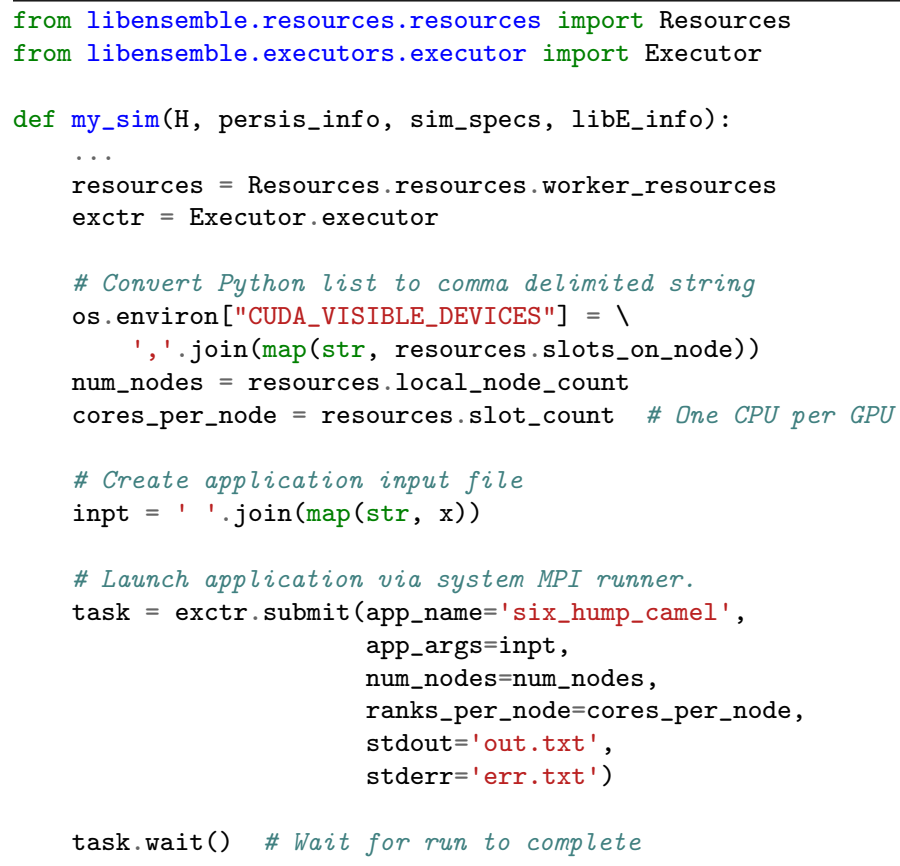

Figure 15: Resources and executor accessed by the user simulator function. 
Both of these approaches are currently being investigated.

Multifidelity ensembles are also currently being explored with collaborators as a use case where simulations resource requirements vary dramatically.

\subsection{Testing at Scale}

The libEnsemble project conducts an extensive set of small-scale unit and regression tests using on-line continuous integration (CI) services (Travis CI and GitHub Actions). However, this is insufficient for testing the interfacing of libEnsemble with HPC systems. Therefore, libEnsemble is regularly tested at large scales with a variety of configurations and test cases on laboratory clusters and leadership computing facilities located throughout the United States. Scaling tests involve ensuring successful installation across systems, MPI initialization, application launches, interoperability with Balsam, and exception handling at large scales. We typically perform this testing manually, but recently we have been actively working to automate the testing process through converting our testing scripts into templates and running our test suite on high-performance CI instances at Argonne National Laboratory.

\subsection{Support for Exascale Machines}

We anticipate supporting ensemble computations on upcoming exascale leadership-class systems such as Aurora and Frontier. In the meantime, we are collaborating closely with scalable application (e.g., WarpX) developers who are looking to perform research on such systems.

\section{Discussion and Outlook}

The use cases provided here represent a narrow slice of near-term exascale-platform applications. We expect that the aims of libEnsemble will evolve to address diverse applications at even larger, more heterogeneous scales. As an example, we note that controlled environment inverse problems such as those arising from 3D tomographic imaging can already saturate current resources [37]. Efficiently accounting for imperfect experimental conditions and understanding key uncertainties necessitate performing not just one expensive inversion but many inversions for ensembles data that are adaptively refined $[38,39]$.

With increasing requirements among users to support diverse resources or machines for ensemble computations, we also anticipate using tools such as Balsam for cross-system application submissions. Users will theoretically be able to launch libEnsemble on one machine and use the executor to launch a subset of applications to a separate machine or set of nodes (each based on a latency that can be statically or dynamically leveraged through an allocation function specification). One such example involves a generator launching machine-learning applications to a GPU machine and training them with data from simulation applications submitted to a CPU machine, such as in Fig. 16.

The ability to use the Balsam-like services to request batch submissions on the fly could also be used for more efficient scheduling in scenarios with highly uncertain time-scales. For instance, libEnsemble could be run in a batch session with a long wall-clock time but just enough resources to accommodate the manager and worker processes. Balsam could request a separate node allocation to accommodate the submitted tasks. Furthermore, new batch submissions (node allocations) could be created as needed, with queued tasks being maintained in the database and pulled from any batch jobs created by the Balsam service. 


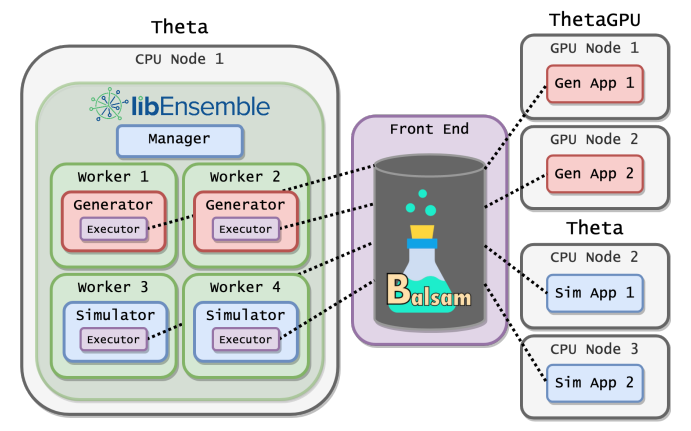

Figure 16: Possible workflow using Balsam for cross-system application submissions. In this example, generators and simulators submit their respective applications separately to ThetaGPU and Theta.

The modular design of libEnsemble means that integration with other pilot systems, runtime environments, and task submission mechanisms can be easily achieved by adding an executor. Also, this modular design has inspired the exploration of the use of a hierarchy of libEnsemble instances, for example, to mitigate manager communication bottlenecks when individual simulator, generator, and allocation functions are especially cheap to evaluate but there is a massive collection of them to be run.

\section{Acknowledgments}

This research was supported in part by the PETSc/TAO activity within the U.S. Department of Energy's (DOE's) Exascale Computing Project (17-SC-20-SC) and by the ComPASS and NUCLEI SciDAC projects within DOE's Office of Science, Advanced Scientific Computing Research under contract number DE-AC02-06CH11357.

We gratefully acknowledge computing resources operated by the Laboratory Computing Resource Center at Argonne National Laboratory and by the DOE Office of Science Argonne and Oak Ridge Leadership Computing Facilities, supported under Contracts DE-AC02-06CH11357 and DE-AC05-00OR22725, respectively.

We are grateful for design help from David Bindel and for inspiration from the PETSc/TAO team. We thank users Lígia Diana Amorim, Moses Chan, Remi Lehe, Nicole Neveu, Matt Plumlee, and Maxence Thévenet for providing problems and data associated with the presented use cases.

\section{References}

[1] S. Hudson, J. Larson, S. M. Wild, D. Bindel, and J.-L. Navarro, "libEnsemble user manual, version 0.7.1," Argonne, Tech report, 2021. [Online]. Available: https: //libensemble.readthedocs.io

[2] S. Balay, S. Abhyankar, M. F. Adams, J. Brown, P. Brune, K. Buschelman, L. Dalcin, A. Dener, V. Eijkhout, W. D. Gropp, D. Karpeyev, D. Kaushik, M. G. Knepley, D. A. May, L. C. McInnes, R. T. Mills, T. Munson, K. Rupp, P. Sanan, B. F. Smith, S. Zampini, H. Zhang, and H. Zhang, "PETSc users manual," Argonne, Tech. Rep. ANL-95/11 - Revision 3.14, 2020. [Online]. Available: https://www.mcs.anl.gov/petsc 
[3] R. T. Mills, M. F. Adams, S. Balay, J. Brown, A. Dener, M. Knepley, S. E. Kruger, H. Morgan, T. Munson, K. Rupp, B. F. Smith, S. Zampini, H. Zhang, and J. Zhang, "Toward performance-portable PETSc for GPU-based exascale systems," arXiv, Preprint 2011.00715, 2020. [Online]. Available: https://arxiv.org/abs/2011.00715

[4] L. Dalcín, R. Paz, M. Storti, and J. D’Elía, "MPI for Python: Performance improvements and MPI-2 extensions," Journal of Parallel and Distributed Computing, vol. 68, no. 5, pp. 655-662, 2008 .

[5] J. Larson and S. M. Wild, "Asynchronously parallel optimization solver for finding multiple minima," Mathematical Programming Computation, vol. 10, no. 3, pp. 303-332, 2018.

[6] — - "A batch, derivative-free algorithm for finding multiple local minima," Optimization and Engineering, vol. 17, pp. 205-228, 2016.

[7] R. Bollapragada, J. Nocedal, D. Mudigere, H.-J. Shi, and P. T. P. Tang, "A progressive batching L-BFGS method for machine learning," in Proc. 35th ICML, J. Dy and A. Krause, Eds., vol. 80. PMLR, 2018, pp. 620-629.

[8] S. Li, T. Ben-Nun, G. Nadiradze, S. Digirolamo, N. Dryden, D. Alistarh, and T. Hoefler, "Breaking (global) barriers in parallel stochastic optimization with wait-avoiding group averaging," IEEE Trans. Parallel Distrib. Syst., pp. 1725-1739, 2020.

[9] P. R. Amestoy, J.-Y. L'Excellent, and G. Moreau, "On exploiting sparsity of multiple righthand sides in sparse direct solvers," SIAM Journal on Scientific Computing, vol. 41, pp. A269-A291, 2019.

[10] M. Gunzburger, T. Iliescu, and M. Schneier, "A Leray regularized ensemble-proper orthogonal decomposition method for parameterized convection-dominated flows," IMA Journal of Numerical Analysis, vol. 40, no. 2, pp. 886-913, 2019.

[11] "The Trilinos Project," available at https://trilinos.github.io.

[12] M. A. Heroux, J. Carter, R. Thakur, J. S. Vetter, L. C. McInnes, J. Ahrens, T. Munson, and J. R. Neely, "ECP software technology capability assessment report," 2020.

[13] M. A. Salim, T. D. Uram, T. Childers, P. Balaprakash, V. Vishwanath, and M. E. Papka, "Balsam: Automated scheduling and execution of dynamic, data-intensive workflows," in Proc. PyHPC 2018, 2018.

[14] R. Bartlett, I. Demeshko, T. Gamblin, G. Hammond, M. Heroux, J. Johnson, A. Klinvex, X. Li, L. C. McInnes, J. D. Moulton, D. Osei-Kuffuor, J. Sarich, B. Smith, J. Willenbring, and U. M. Yang, "xSDK foundations: Toward an extreme-scale scientific software development kit," Supercomputing Frontiers and Innovations: an International Journal, vol. 4, no. 1, pp. 69-82, 2017.

[15] V. Balasubramanian, A. Treikalis, O. Weidner, and S. Jha, "Ensemblemd toolkit: Scalable and flexible execution of ensembles of molecular simulations," CoRR, vol. abs/1602.00678, 2016. [Online]. Available: http://arxiv.org/abs/1602.00678

[16] "ExaWorks," available at https://github.com/exaworks. 
[17] Y. Babuji, A. Woodard, Z. Li, D. S. Katz, B. Clifford, R. Kumar, L. Lacinski, R. Chard, J. Wozniak, I. Foster, M. Wilde, and K. Chard, "Parsl: Pervasive parallel programming in python," in Proc. HPDC19, 2019.

[18] D. H. Ahn, J. Garlick, M. Grondona, D. Lipari, B. Springmeyer, and M. Schulz, "Flux: A next-generation resource management framework for large HPC centers," in ICPP Works., 2014, pp. 9-17.

[19] J. Jenkins, J. Dinan, P. Balaji, T. Peterka, N. F. Samatova, and R. Thakur, "Processing MPI derived datatypes on noncontiguous GPU-resident data," IEEE Trans. Parallel Distrib. Syst., vol. 25, no. 10, pp. 2627-2637, 2014.

[20] D. Eriksson, D. Bindel, and C. Shoemaker, "pySOT and POAP: An event-driven asynchronous framework for surrogate optimization," arXiv, Preprint 1908.00420, 2019. [Online]. Available: https://arxiv.org/pdf/1908.00420

[21] S. G. Johnson, "The NLopt nonlinear-optimization package," 2021. [Online]. Available: http://github.com/stevengj/nlopt

[22] A. Adelmann, P. Calvo, M. Frey, A. Gsell, U. Locans, C. Metzger-Kraus, N. Neveu, C. Rogers, S. Russell, S. Sheehy, J. Snuverink, and D. Winklehner, "OPAL a versatile tool for charged particle accelerator simulations," arXiv, Preprint, 2019. [Online]. Available: https://arxiv.org/abs/1905.06654

[23] R. Lemons, N. Neveu, J. Duris, A. Marinelli, C. Durfee, and S. Carbajo, "Dispersion-controlled temporal shaping of picosecond pulses via non-colinear sum frequency generation," arXiv, Preprint, 2020. [Online]. Available: https://arxiv.org/abs/2012.00957

[24] S. Steinke, J. van Tilborg, C. Benedetti, C. G. R. Geddes, C. B. Schroeder, J. Daniels, K. K. Swanson, A. J. Gonsalves, K. Nakamura, N. H. Matlis, B. H. Shaw, E. Esarey, and W. P. Leemans, "Multistage coupling of independent laser-plasma accelerators," Nature, vol. 530, no. 7589 , pp. 190-193, 2016.

[25] J.-L. Vay, A. Almgren, J. Bell, L. Ge, D. Grote, M. Hogan, O. Kononenko, R. Lehe, A. Myers, C. Ng, J. Park, R. Ryne, O. Shapoval, M. Thévenet, and W. Zhang, "Warp-X: A new exascale computing platform for beam-plasma simulations," Nuclear Instruments and Methods in Physics Research Section A: Accelerators, Spectrometers, Detectors and Associated Equipment, vol. 909, pp. 476-479, 2018.

[26] A. Myers, A. Almgren, L. D. Amorim, J. Bell, L. Fedeli, L. Ge, K. Gott, D. P. Grote, M. Hogan, A. Huebl, R. Jambunathan, R. Lehe, C. Ng, M. Rowan, O. Shapoval, M. Thévenet, J. L. Vay, H. Vincenti, E. Yang, N. Zaïm, W. Zhang, Y. Zhao, and E. Zoni, "Porting WarpX to GPU-accelerated platforms," arXiv, Preprint 2101.12149, 2021. [Online]. Available: https://arxiv.org/abs/2101.12149

[27] M. J. D. Powell, "The BOBYQA algorithm for bound constrained optimization without derivatives," University of Cambridge, Tech. Rep. DAMTP 2009/NA06, 2009. [Online]. Available: http://www.damtp.cam.ac.uk/user/na/NA_papers/NA2009_06.pdf

[28] R. B. Gramacy, Surrogates: Gaussian Process Modeling, Design and Optimization for the Applied Sciences. Chapman Hall/CRC, 2020. 
[29] E. Baker, P. Barbillon, A. Fadikar, R. B. Gramacy, R. Herbei, D. Higdon, J. Huang, L. R. Johnson, P. Ma, A. Mondal, B. Pires, J. Sacks, and V. Sokolov, "Analyzing stochastic computer models: A review with opportunities," arXiv, Preprint 2002.01321, 2020. [Online]. Available: https://arxiv.org/abs/2002.01321

[30] M. Binois, J. Huang, R. B. Gramacy, and M. Ludkovski, "Replication or exploration? Sequential design for stochastic simulation experiments," Technometrics, vol. 61, no. 1, pp. 7-23, 2018 .

[31] N. Bliznyuk, D. Ruppert, C. A. Shoemaker, R. G. Regis, S. M. Wild, and P. Mugunthan, "Bayesian calibration of computationally expensive models using optimization and radial basis function approximation," Journal of Computational and Graphical Statistics, vol. 17, no. 2, pp. 270-294, 2008.

[32] M. Plumlee, O. Sürer, and S. M. Wild, Surmise Users Manual, v. 0.1.0, 2021. [Online]. Available: https://surmise.readthedocs.io

[33] M. Plumlee, "Computer model calibration with confidence and consistency," Journal of the Royal Statistical Society: Series B, vol. 81, no. 3, pp. 519-545, 2019.

[34] M. D. Morris, T. J. Mitchell, and D. Ylvisaker, "Bayesian design and analysis of computer experiments: Use of derivatives in surface prediction," Technometrics, vol. 35, no. 3, pp. 243255, 1993.

[35] M. Karo, R. Lagerstrom, M. Kohnke, and C. Albing, "The application level placement scheduler," in Cray User Group, 2006, pp. 1-7.

[36] C. Fuson, "OLCF Training Archive launching multiple jsruns," ORNL, Tech report, 2020. [Online]. Available: https://www.olcf.ornl.gov/wp-content/uploads/2019/12/Jsrun-Multi.pdf

[37] M. Hidayetoğlu, T. Bicer, S. G. de Gonzalo, B. Ren, V. De Andrade, D. Gursoy, R. Kettimuthu, I. T. Foster, and W.-m. W. Hwu, "Petascale XCT: 3D image reconstruction with hierarchical communications on multi-GPU nodes," in Proc. SC20. IEEE, 2020.

[38] M. Kotsi, A. Malcolm, and G. Ely, "Uncertainty quantification in time-lapse seismic imaging: a full-waveform approach," Geophysical Journal International, vol. 222, no. 2, pp. 1245-1263, 2020 .

[39] A. Austin, Z. W. Di, S. Leyffer, and S. M. Wild, "Simultaneous sensing error recovery and tomographic inversion using an optimization-based approach," SIAM Journal on Scientific Computing, vol. 41, no. 3, pp. B497-B521, 2019. 
The submitted manuscript has been created by UChicago Argonne, LLC, Operator of Argonne National Laboratory ("Argonne"). Argonne, a U.S. Department of Energy Office of Science laboratory, is operated under Contract No. DE-AC02-06CH11357. The U.S. Government retains for itself, and others acting on its behalf, a paid-up nonexclusive, irrevocable worldwide license in said article to reproduce, prepare derivative works, distribute copies to the public, and perform publicly and display publicly, by or on behalf of the Government. The Department of Energy will provide public access to these results of federally sponsored research in accordance with the DOE Public Access Plan. http://energy.gov/downloads/doe-public-accessplan. 